Preprint typeset in JHEP style - HYPER VERSION

\title{
Parikh-Wilczek Tunneling from Noncommutative Higher Dimensional Black Holes
}

\author{
Kourosh Nozari ${ }^{a, b}$ and S. Hamid Mehdipour ${ }^{a, c}$ \\ ${ }^{a}$ Department of Physics, Faculty of Basic Sciences, University of Mazandaran, \\ P. O. Box 47416-95447, Babolsar, IRAN. \\ ${ }^{b}$ Research Institute for Astronomy and Astrophysics of Maragha, \\ P. O. Box 55134-441, Maragha, IRAN. \\ ${ }^{c}$ Islamic Azad University, Lahijan Branch, \\ P. O. Box 1616, Lahijan, IRAN. \\ E-mail: knozari@umz.ac.ir, h.mehdipour@umz.ac.in
}

\begin{abstract}
We study tunneling of massless and massive particles through the smeared quantum horizon of the extra-dimensional Schwarzschild black holes. The emission rate of the particles' tunneling is modified by noncommutativity effects in a bulk spacetime of dimension $d$. The issues of information loss and possible correlations between emitted particles are discussed. We show that even by considering both noncommutativity and braneworld effects, there is no correlation between different modes of evaporation at least at late-time and within approximations used in the calculations. However, incorporation of quantum gravity effects such as modification of the standard dispersion relation or generalization of the Heisenberg uncertainty principle, leads to the correlation between emitted particles. Although time-evolution of these correlations is not trivial, a part of information coming out of the black hole can be preserved in these correlations. On the other hand, as a well-known result of spacetime noncommutativity, a part of information may be preserved in a stable black hole remnant.
\end{abstract}

KEywords: Black Holes, Classical Theories of Gravity. 


\section{Contents}

1. Introduction

2. Noncommutativity in the Presence of Extra Dimensions 2

3. Particles' Tunneling Near the Smeared Quantum Horizon 8

4. Quantum Gravity as a Source of Correlations 13

5. Summary 17

A. 17

\section{Introduction}

Radiation spectrum of an evaporating black hole which is almost similar to black body radiation spectrum, can be described by a characteristic temperature dubbed Hawking temperature. By adopting a quantum field theoretical approach in curved spacetime, this characteristic temperature is given by $T_{H}=\frac{\hbar c^{3} \kappa}{2 \pi k_{B} G}$, where $\kappa$ is the surface gravity that demonstrates the strength of the gravitational field near the black hole surface [1]. Unfortunately, Hawking's approach leads to a non-unitarity of quantum theory which maps a pure state to a mixed state as a result of purely thermal nature of the spectrum. A few years ago, Parikh and Wilczek [2] suggested a new method based on null-geodesics to extract the Hawking radiation via tunneling across the event horizon. This method modifies the form of the black hole radiation spectrum that is due to inclusion of back-reaction effects. The tunneling process clarifies that the modified radiation spectrum is not precisely thermal and this leads to the unitarity of underlying quantum theory [3]. Nevertheless, the form of the correction is not sufficient by itself to recover information because of its failure to have correlations between the tunneling probability of different modes in the black hole radiation spectrum [4]. Recently, Nicolini, Smailagic and Spallucci (NSS) [5], in a new conceptual approach to space noncommutativity, have shown that black hole evaporation process should be terminated when black hole reaches to a minimal mass, i.e. black hole remnant (for a review see [6]). This minimal mass is a consequence of existence of minimal observable length [7, 8, 9, 10, 11]. The NSS viewpoint on coordinate noncommutativity is carried out by the Gaussian distribution of coherent states and is consistent with Lorentz invariance, unitarity and UV-finiteness of quantum field theory [12]. Moreover, noncommutativity of spacetime is an inherent property of the manifold by itself even if there is no gravity. In this framework, some kind of divergencies which arise in general relativity 
and black hole physics, can be removed also. In a recent paper [13], we have studied the Parikh-Wilczek tunneling through the quantum horizon of a Schwarzschild black hole in noncommutative (NC) spacetime. We have shown that, in the proposed NC setup there is no correlation between different modes of the radiation which reflects the fact that information does not come out continuously during the evaporation process, at least at late-time. The fundamental motivation of the present paper is to find an alternative framework to recover the lost information in the black hole evaporation process (see [14] for comprehensive reviews on existing approaches to resolution of black hole information paradox). This alternative framework can be identified with the correlations between different modes of evaporation. Another question that we focus on is the possible impact of braneworld scenarios and correlation between different emitted modes of black hole evaporation. In other words, we want to know whether inclusion of extra spatial dimensions in the framework of extra dimensional scenarios can create correlation between different modes or not. This is an important question which is considered here via the tunneling framework of Parikh and Wilczek. With these motivations, we apply back-reaction and noncommutativity effects to study black hole evaporation process in a bulk spacetime of dimension $d$. Recently, we have shown that incorporating quantum gravity effects (such as Generalized Uncertainty Principle (GUP) [7, 8, 9, 10, 11] (see also [15, 16]) and Modified Dispersion Relations (MDR) [17, 18, 19]) in the black hole evaporation process has the capability to produce correlations between different emitted modes [20]. Although the time evolution of these correlations are not obvious and deserve further investigations, the possibility of realizing these correlations itself is an important step toward the resolution of black hole information paradox. Here we generalize this achievement to our extra dimensional framework and discuss some related issues.

The paper is organized as follows: In Section 2 we construct a noncommutative framework in the presence of extra dimensions and we study evaporation of Schwarzschild black hole in this setup. In Section 3, a detailed calculation of quantum tunneling near the

smeared quantum horizon is provided. We calculate tunneling probability of massless and massive particles through the quantum horizon. The problem of lost information and possible correlations between emitted particles are investigated. The capability of the black hole evaporation process to produce the correlations between different emitted modes via incorporation of quantum gravity effects is investigated in Section 4 . Summary and conclusions are presented in Section 5 .

\section{Noncommutativity in the Presence of Extra Dimensions}

Noncommutative quantum field theories (NCQFTs) have attracted a lot of attentions during last two decades [21] (see also [22] for a purely phenomenological viewpoint on this issue). There are also a lot of attempts to possible realization of these effects on experimental grounds 23]. The NC spacetime coordinates, in their simplest form, are deformations of usual spacetime coordinates (with an arbitrary number, $d$ of spacetime dimensions) in which operators $X^{A}$ representable by the Hermitian operators $\hat{X}^{A}$, characterize a nonvan- 
ishing commutation relation as follows

$$
\left[\hat{X}^{A}, \hat{X}^{B}\right]=i \theta^{A B}=i \frac{C^{A B}}{\Lambda_{N C}^{2}},
$$

where $\Lambda_{N C}$ is a characteristic energy or inverse length scale related to the NC effects and $C^{A B}\left(\theta^{A B}\right)$ is a dimensionless, real and anti-symmetric matrix, whose elements are constant and are assumed to be of the order of one. The physical explanation of the $\theta^{A B}$ is the smallest fundamental cell of observable area in the $A B$-plane, in the same way as Planck constant $\hbar$ explains the smallest fundamental cell of observable phase space in quantum mechanics. The scale $\Lambda_{N C}$, possibly and most reasonably is of the order of Planck scale $M_{P l}$. This is supported by the fact that most of the phenomenological studies of the NC models have presumed that $\Lambda_{N C}$ cannot lie far above the TeV scale [22, 23]. Since the fundamental Planck scale in models with large extra dimensions (LEDs) becomes as small as a $\mathrm{TeV}$ in order to solve the hierarchy problem [24, 25]; it is possible to set the NC effects in a $\mathrm{TeV}$ regime.

The framework of NCQFT is achieved by substitution of ordinary products in commutative theory with Moyal $\star$-products [26]. There exist many formulations of NCQFT based on the Moyal $\star$-product that lead to failure in resolving of some important problems, such as Lorentz invariance breaking, loss of unitarity and UV divergences of quantum field theory. Unfortunately, no flawless and completely convincing theory of noncommutativity exists yet. Recently, the authors in Ref. [12] explained a fascinating model of noncommutativity, the coordinate coherent states approach, that can be free from the problems mentioned above. In this approach, General Relativity in its usual commutative form as described by the Einstein-Hilbert action remains applicable. If noncommutativity effects can be treated in a perturbative manner, then this is defensible, at least to a good approximation. Indeed, the authors in Ref. [27] have shown that the leading noncommutativity corrections to the form of the Einstein-Hilbert action are at least second order in the noncommutativity parameter $\theta$. The generalization of the QFT by noncommutativity based on coordinate coherent state formalism is also interestingly curing the short distance behavior of pointlike structures [5, 12] (see also [28, 29]). In this method, the particle mass $M$, instead of being quite localized at a point, is described by a smeared structure throughout a region of linear size $\sqrt{\theta}$. In other words, we shall smear the point mass Dirac-delta function utilizing a Gaussian function of finite width. So, instead of a delta function distribution, the mass density distribution of a static, spherically symmetric, particle-like gravitational source in $d$-dimensional bulk spacetime will be given by a Gaussian distribution of minimal width $\sqrt{\theta}$ as follows ${ }^{1} 29$

$$
\rho_{\theta}(r)=\frac{M}{(4 \pi \theta)^{\frac{d-1}{2}}} e^{-\frac{r^{2}}{4 \theta}} .
$$

The line element which solves Einstein's field equations $G_{B A}=8 \pi G_{d} T_{B A}$ in the presence of smeared mass sources can be obtained as

$$
d s^{2}=-\left(1-\frac{2 \mathcal{M}_{\theta}}{r^{d-3}}\right) d t^{2}+\left(1-\frac{2 \mathcal{M}_{\theta}}{r^{d-3}}\right)^{-1} d r^{2}+r^{2} d \Omega_{(d-2)}^{2},
$$

\footnotetext{
${ }^{1}$ Throughout the rest of this paper, natural units are used so that $\hbar=c=k_{B}=1$.
} 
with

$$
\mathcal{M}_{\theta}=\frac{m}{\Gamma\left(\frac{d-1}{2}\right)} \int_{0}^{\frac{r^{2}}{4 \theta}} d t e^{-t} t^{\left(\frac{d-3}{2}\right)}, m=\frac{8 \pi G_{d} M}{(d-2) \Omega_{(d-2)}} .
$$

Where $d \Omega_{(d-2)}^{2}$ is the line element on the $(d-2)$-dimensional unit sphere and $d$ is spacetime dimensionality. The volume of the $(d-2)$-dimensional unit sphere is given by

$$
\Omega_{(d-2)}=\frac{2 \pi^{\frac{d-1}{2}}}{\Gamma\left(\frac{d-1}{2}\right)} .
$$

$G_{d}$ is gravitational constant in $d$-dimensional spacetime which in ADD [25] model is given by

$$
G_{d}=\frac{(2 \pi)^{d-4}}{\Omega_{d-2}} M_{P l}^{2-d},
$$

where $M_{P l}$ is the $d$-dimensional Planck mass and there is an effective 4-dimensional Newton constant related to $M_{P l}$ by

$$
M_{P l}^{2-d}=4 \pi G_{4} R^{d-4},
$$

where $R$ is the common radius of all $(d-4)$-toroidally compactified extra dimensions. We note that in this work, the conventions for definition of the fundamental Planck scale $M_{P l}$ are the same as which have been used by ADD (and also by authors of Ref. [30]). The metric (2.3) smoothly interpolates between de Sitter core around the origin and an ordinary Schwarzschild geometry far away from the origin [5, 29]. On the other hand, the curvature singularity at the point $r=0$ is eliminated by noncommutativity as an inherent property of the manifold. In this situation, a regular de Sitter vacuum state will be formed accounting for the effect of the $\mathrm{NC}$ coordinates fluctuations at short distances. Also, this scenario tends to usual Schwarzschild spacetime at large distances. In the limit $\theta \rightarrow 0$, one recovers the complete Gamma function, $\int_{0}^{\frac{r^{2}}{4 \theta}} d t e^{-t} t^{\left(\frac{d-3}{2}\right)} \rightarrow \Gamma\left(\frac{d-1}{2}\right)$, and $\mathcal{M}_{\theta} \rightarrow m$ as expected. Taking a 4-dimensional viewpoint in the commutative case, one can simply obtain $\mathcal{M}_{\theta}=G_{4} M$. Then the NC (modified) Schwarzschild solution reduces to the commutative (ordinary) case. Depending on the different values of $M, d$ and $M_{P l}$ and within a numerical procedure, the metric (2.3) displays three possible causal structure [5, 13, 29, 31] (see also [32, 33]): 1It is possible to have two distinct horizons (Non-extremal Black Hole), 2- It is possible to have one degenerate horizon (Extremal Black Hole), and finally 3- It is impossible to have horizon at all.

The radiating behavior of such a modified Schwarzschild black hole can now be easily investigated by plotting the temporal component of the metric, $g_{00}$, versus the radius $r$ for an extremal black hole with different values of $M$ and $d$. This has been shown in Fig. (11). As this figure shows, the coordinate noncommutativity leads to the existence of a minimal non-zero mass in which black hole (due to Hawking radiation) can shrink to ${ }^{2}$. It should be emphasized that based on our computations, if we set $d=11$, we find

\footnotetext{
${ }^{2}$ For simplicity of numerical calculations, we assume $\theta=1$. Naturally $\frac{1}{\sqrt{\theta}}\left(\Lambda_{N C}\right)$ points out the energy threshold beyond which a particle moves into the deformed spacetime in which quantum gravity effects become important.
} 


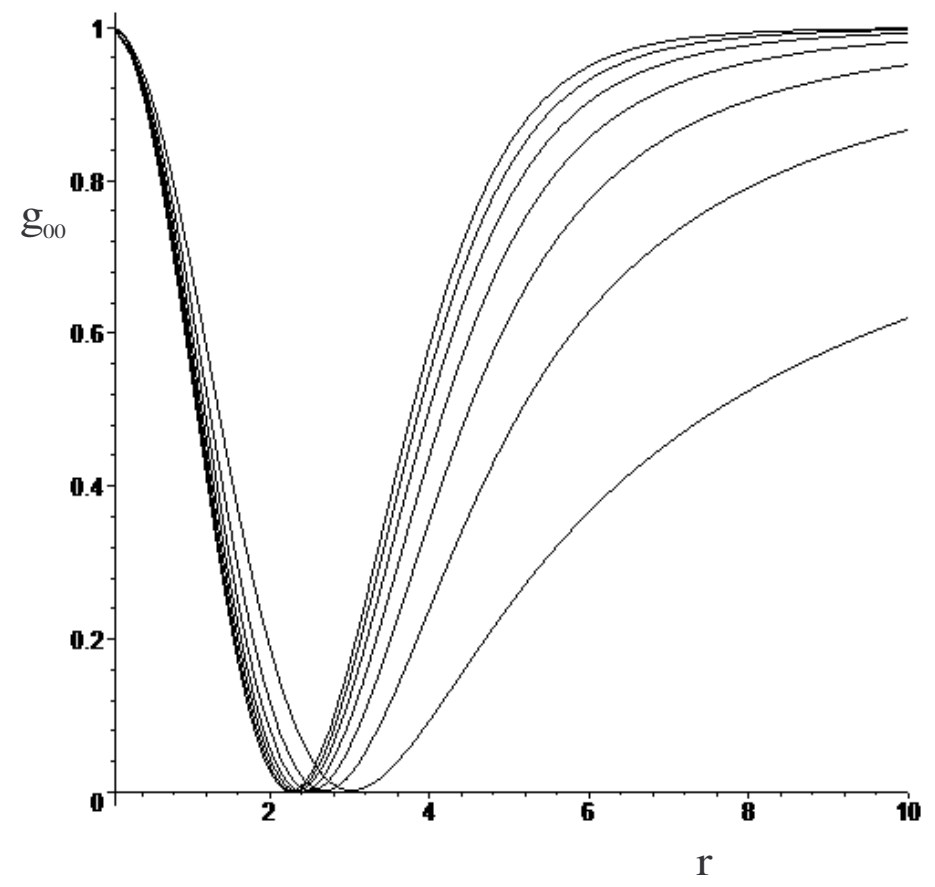

Figure 1: $g_{00}$ versus the radius $r$ for different values of black hole mass $M$ and different number of spacetime dimensions, $d$. The figure shows the possibility of having extremal configuration with one degenerate event horizon (Extremal Black Hole) at $M=M_{0}$. This shows the existence of a minimal non-zero mass that black hole shrinks to. On the right-hand side of the figure, curves are marked from bottom to top by $d=4$ to $d=10$ (with $M_{P l}=1$ ).

Table 1: Minimal non-zero mass of the black hole (remnant mass) for different number of spacetime dimensions.

\begin{tabular}{|c||c|}
\hline \multicolumn{2}{|c|}{ Extremal Black Hole $; M_{P l}=1 \mathrm{TeV}$} \\
\hline$d$ & Minimal Mass $(\mathrm{TeV})$ \\
\hline 4 & $M=M_{0} \approx 1.9$ \\
\hline 5 & $M=M_{0} \approx 3.94$ \\
\hline 6 & $M=M_{0} \approx 5.43$ \\
\hline 7 & $M=M_{0} \approx 5.77$ \\
\hline 8 & $M=M_{0} \approx 5.08$ \\
\hline 9 & $M=M_{0} \approx 3.88$ \\
\hline 10 & $M=M_{0} \approx 2.65$ \\
\hline 11 & $M=M_{0} \approx 1.64$ \\
\hline
\end{tabular}

$M=M_{0} \approx 1.64 \mathrm{TeV}$, i.e. the minimum value of black hole mass reduces to a value less than its value for $d=4$ (see Table 1). Therefore, in theories with large extra dimensions, if the number of spacetime dimensions becomes sufficiently great, e.g. $d \geq 11$ with a sufficiently small NC parameter $\left(\Lambda_{N C} \sim M_{P l} \sim 1 \mathrm{TeV}\right)$, then the noncommutativity effect can enhance the possible formation and detection of black holes in $\mathrm{TeV}$-scale collisions at the $\mathrm{LHC}^{3}$ 34.

\footnotetext{
${ }^{3} \mathrm{~A}$ micro-black hole in theories with large extra dimensions can be produced at the large hadron collider (LHC) just in the situation that $E_{c m}>M_{0}$, where $E_{c m}$ is parton-parton center-of-mass energy which is equal to $14 \mathrm{TeV}$.
} 
The event horizon radius, $r_{H}$, can be obtained from the equation $g_{00}\left(r_{H}\right)=0$, which gives

$$
1-\frac{2 \mathcal{M}_{\theta}\left(r_{H}\right)}{r_{H}^{d-3}}=0
$$

with

$$
\begin{aligned}
\mathcal{M}_{\theta}\left(r_{H}\right) \equiv & 4 \pi G_{d} M 2^{3-d}\left(\frac{r_{H}^{2}}{\theta}\right)^{\frac{d-3}{2}}\left\{2^{\frac{d-3}{2}} r_{H}^{4}\left(\frac{r_{H}^{2}}{\theta}\right)^{-\frac{d+1}{4}} e^{-\frac{r_{H}^{2}}{8 \theta}} \mathcal{W}\left(\frac{d+1}{4}, \frac{d+3}{4}, \frac{r_{H}^{2}}{4 \theta}\right)\right. \\
& {\left[\left(\frac{d-1}{2}\right) \theta^{2}(1+d)(d+3)\right]^{-1}+\left(\frac{r_{H}^{2}}{2 \theta}+1+d\right)\left(\frac{r_{H}^{2}}{\theta}\right)^{-\frac{d+1}{4}} 2^{\frac{d+1}{2}} e^{-\frac{r_{H}^{2}}{8 \theta}} } \\
& \left.\mathcal{W}\left(\frac{d+5}{4}, \frac{d+3}{4}, \frac{r_{H}^{2}}{4 \theta}\right)\left[\left(\frac{d-1}{2}\right)(1+d)\right]^{-1}\right\}\left((d-2) \pi^{\frac{d-1}{2}}\right)^{-1} .
\end{aligned}
$$

Where $\mathcal{W}(\mu, \nu, z)$ shows the Whittaker function defined in terms of the hypergeometric functions as follows

$$
\mathcal{W}(\mu, \nu, z)=\text { hypergeom }\left(\nu-\mu+\frac{1}{2}, 2 \nu+1, z\right) e^{-\frac{z}{2}} z^{\nu+\frac{1}{2}} .
$$

Analytical solution of the Eq. (2.8) for $r_{H}$ in a closed form is impossible, but we can approximately solve it by setting $r_{H}=2 M$ into the lower incomplete Gamma function of the Eq. (2.4) to find ${ }^{4}$

$$
\begin{gathered}
r_{H}=\left(2 \mathcal{M}_{\theta}\left(r_{H}\right)\right)^{\frac{1}{d-3}} \approx\left\{\frac { 8 \pi G _ { d } M ^ { d } } { \theta ^ { \frac { d - 3 } { 2 } } } \left[4 \frac{M^{-\frac{d-3}{2}}}{\theta^{-\frac{d+1}{4}}} e^{-\frac{M^{2}}{2 \theta}} \mathcal{W}\left(\frac{d+1}{4}, \frac{d+3}{4}, \frac{M^{2}}{\theta}\right)\right.\right. \\
\left(\left(\frac{d-1}{2}\right) \theta(1+d)(d+3)\right)^{-1}+\left(2 \frac{M^{2}}{\theta}+1+d\right) \theta\left(\frac{M^{2}}{\theta}\right)^{-\frac{d+1}{4}} e^{-\frac{M^{2}}{2 \theta}} \\
\left.\left.\mathcal{W}\left(\frac{d+5}{4}, \frac{d+3}{4}, \frac{M^{2}}{\theta}\right)\left(\left(\frac{d-1}{2}\right) M^{2}(1+d)\right)^{-1}\right]\left((d-2) \pi^{\frac{d-1}{2}} \theta\right)^{-1}\right\}^{\frac{1}{d-3}} .
\end{gathered}
$$

For very large masses, one can easily recover the classical Schwarzschild radius in a spacetime with $d$ dimensions, $r_{H} \approx(2 m)^{\frac{1}{d-3}}$.

Now we calculate Hawking temperature of noncommutative black hole by investigating its radiating behavior. By definition,

$$
T_{H}=\left.\frac{1}{4 \pi} \frac{d g_{00}}{d r}\right|_{r=r_{H}} .
$$

Black hole temperature for some arbitrary number of spacetime dimensions can be calculated as follows

$$
T_{4}=G_{4} M\left[\frac{\mathcal{E}\left(\frac{r_{H}}{2 \sqrt{\theta}}\right)}{2 \pi r_{H}^{2}}-\frac{r_{H} e^{-\frac{r_{H}^{2}}{4 \theta}}}{4(\pi \theta)^{\frac{3}{2}}}\left(1+\frac{2 \theta}{r_{H}^{2}}\right)\right]
$$

\footnotetext{
${ }^{4}$ We will use this approximation in the next section too.
} 


$$
\begin{gathered}
T_{5}=G_{5} M\left[\frac{4}{3 \pi^{2} r_{H}^{3}}-\frac{r_{H} e^{-\frac{r_{H}^{2}}{4 \theta}}}{12(\pi \theta)^{2}}\left(1+\frac{4 \theta}{r_{H}^{2}}+\frac{16 \theta^{2}}{r_{H}^{4}}\right)\right], \\
T_{6}=G_{6} M\left[\frac{9 \mathcal{E}\left(\frac{r_{H}}{2 \sqrt{\theta}}\right)}{8 \pi^{2} r_{H}^{4}}-\frac{r_{H} e^{-\frac{r_{H}^{2}}{4 \theta}}}{32(\pi \theta)^{\frac{5}{2}}}\left(1+\frac{6 \theta}{r_{H}^{2}}+\frac{36 \theta^{2}}{r_{H}^{4}}\right)\right], \\
T_{7}=G_{7} M\left[\frac{32}{10 \pi^{3} r_{H}^{5}}-\frac{r_{H} e^{-\frac{r_{H}^{2}}{4 \theta}}}{80(\pi \theta)^{3}}\left(1+\frac{8 \theta}{r_{H}^{2}}+\frac{64 \theta^{2}}{r_{H}^{4}}+\frac{256 \theta^{3}}{r_{H}^{6}}\right)\right],
\end{gathered}
$$

and so on. $\mathcal{E}(x)$ shows the Gauss Error Function defined as follows

$$
\mathcal{E}(x) \equiv \frac{2}{\sqrt{\pi}} \int_{0}^{x} e^{-t^{2}} d t .
$$

While for all even values of $d$ Hawking temperature can be expressed in terms of the Gauss error function, for odd values of $d$ Hawking temperature has a closed analytical form. For very large distances (or commutative case), the function $\mathcal{E}\left(\frac{r_{H}}{2 \sqrt{\theta}}\right)$ for even values of $d$ tends to unity and other terms on the right hand side will be vanishing exponentially. Therefore, one recovers the classical Hawking temperature in $d$ dimensions

$$
T_{H}=2\left(\frac{d-3}{d-2}\right) \pi^{-\frac{d-1}{2}} G_{d} M \Gamma\left(\frac{d-1}{2}\right) r_{H}^{2-d} .
$$

In this situation, if we want to obtain the simple $d$-dependent form of the noncommutative Hawking temperature, then it can be approximated by utilizing the Eq. (2.9) as follows

$$
T_{H}=\frac{d-3}{4 \pi r_{H}} .
$$

We will come back to this relation later in this paper.

It should be stressed that, if instead of distribution (2.2), we adopt other kinds of probability distributions, only the smeared mass distribution $\mathcal{M}_{\theta}$ will change and all general properties will be the same as above. For example, we consider a Lorentzian distribution of smeared matter as follows ${ }^{5}$

$$
\rho_{\theta^{\prime}}(r)=\frac{M \sqrt{\theta^{\prime}}}{\pi^{2}\left(r^{2}+\theta^{\prime}\right)^{\frac{d}{2}}} .
$$

The smeared mass distribution is now given by

$$
\mathcal{M}_{\theta^{\prime}}=\frac{2 m(d-2) ! !}{\pi(d-3) ! !} \int_{0}^{\frac{r}{\sqrt{\theta^{\prime}}}} \frac{t^{d-2}}{\left(1+t^{2}\right)^{\frac{d}{2}}} d t .
$$

In the limit of $\theta^{\prime} \rightarrow 0$, we obtain $\mathcal{M}_{\theta^{\prime}} \rightarrow m$. The Lorentzian smeared mass, $\mathcal{M}_{\theta^{\prime}}$, has limiting properties similar to Gaussian one and therefore can be compared with Gaussian smeared mass distribution, $\mathcal{M}_{\theta}$. Thus, most of the outcomes that we obtained for Gaussian

\footnotetext{
${ }^{5}$ Generally, the noncommutativity parameter $\theta^{\prime}$ appeared in Eq. (2.17) is not exactly the same as $\theta$ appeared in Eq. (2.2).
} 
profile, stay applicable if we pick out a different form of the probability distribution of smeared matter ${ }^{6}$ (see also [13, 29]).

As an important remark, we note that some authors have studied black hole thermodynamics in the NC framework adopting a coordinate noncommutativity against coherent state approach (see [35] and references therein). A question then arises: what is the difference between these two approaches? The standard way to handle NC problems is based on Moyal $\star$-product. That means to use complex number commuting coordinates and shift noncommutativity in the product between functions. This is mathematically valid, but it is physically useless since any model written in terms of $\star$-product, even the simplest field theory, is nonlocal and it is not obvious how to handle nonlocal quantum field theory. One suggested approach is perturbation in the $\theta$ parameter [36]. This is physically reasonable due to the fact that once expanded up to a given order in $\theta$, the resulting field theory becomes local. The smeared picture of particles based on coordinate coherent states defines complex number coordinates as quantum mean values of the original noncommuting ones between coordinate coherent states [12]. In other words, in this setup one can see commuting coordinates as classical limit (in the quantum mechanical sense) of the noncommuting ones. In this framework, the emergent semiclassical geometry keeps memory of its origin. For example, free propagation of a point-like object is described by a minimal width Gaussian wave-packet as has been considered in our setup. So, the difference between two approaches lies in the definition of quantum field theoretical propagators.

\section{Particles' Tunneling Near the Smeared Quantum Horizon}

Now we provide a detailed calculation of quantum tunneling from the NC black hole event horizon. To portray the NC quantum tunneling procedure, where a particle moves in dynamical geometry and passes through the horizon without singularity on the path, we should take advantage of a coordinates system that, unlike Schwarzschild coordinates, is not singular at the horizon. A convenient choice in this regard is Painlevé coordinate transformation [37] which is obtained by definition of a new NC time coordinate

$$
d t=d t_{s}+\frac{\sqrt{2 \mathcal{M}_{\theta} r^{d-3}}}{r^{d-3}-2 \mathcal{M}_{\theta}} d r
$$

where $t_{s}$ is the Schwarzschild time coordinate. Note that only Schwarzschild time coordinate is transformed, while radial coordinate and angular coordinates remain unchanged. Now the NC Painlevé-Schwarzschild metric in spacetime with $d$ dimensions takes the following form

$$
d s^{2}=-\left(1-\frac{2 \mathcal{M}_{\theta}}{r^{d-3}}\right) d t^{2}+2 \sqrt{\frac{2 \mathcal{M}_{\theta}}{r^{d-3}}} d t d r+d r^{2}+r^{2} d \Omega_{(d-2)}^{2} .
$$

\footnotetext{
${ }^{6}$ The difference between various forms of smeared mass distributions is considerable around $M<M_{0}$ where there are sensitivity to noncommutative effects and detailed form of the matter distribution. Therefore, we focus on situations that the condition $M \geq M_{0}$ holds.
} 
The metric in these new coordinates is stationary and non-static. In addition, neither coordinate nor intrinsic singularities are present. Radial null geodesics (the equation of motion for massless particles) in this geometry are as follows

$$
\dot{r} \equiv \frac{d r}{d t}= \pm 1-\sqrt{\frac{2 \mathcal{M}_{\theta}}{r^{d-3}}}
$$

where the upper (lower) sign corresponds to an outgoing (ingoing) null geodesic respectively. If we assume $t$ increases in the direction of future, then the above equations will be corrected by the particle's self-gravitation effect. Kraus and Wilczek [38] scrutinized the motion of particles in the $s$-wave as spherical massless shells in dynamical geometry and expanded self-gravitating shells in Hamiltonian gravity (see also [39]). Supplementary elaborations was accomplished by Parikh and Wilczek [2]. In this manner, we are going to develop their framework to the NC coordinate coherent states in a model universe with extra-dimensions.

We set the total ADM mass $(M)$ of the spacetime to be fixed, and permit the hole mass to fluctuate. Also, we consider the reaction of the background geometry to an emitted quantum of energy $E$ which moves on the geodesics of a spacetime with $M$ replaced by $M-E$. In this manner, we should replace $M$ by $M-E$ in Eqs. (3.1, 3.2, and 3.3). The geodesic equation of motion, Eq. (3.3), can be written as

$$
\dot{r}= \pm 1-\sqrt{\frac{2 \mathcal{M}_{\theta}(M-E)}{r^{d-3}}} .
$$

Since the characteristic wavelength of the radiation near the horizon is always small due to infinite blue-shift, the wave-number approaches infinity. Hence the WKB approximation is valid near the horizon. In the WKB limit, the tunneling probability or emission rate for the classically forbidden trajectories as a function of imaginary part of the particle action at stationary phase takes the following form

$$
\Gamma \sim \exp (-2 \operatorname{Im} I)
$$

To compute the imaginary part of the action, we consider a spherical shell of massless particles that move on radial null geodesics. Since the radial null geodesics can be interpreted as $s$-waves outgoing positive energy particles which cross the horizon outward from $r_{i n}$ to $r_{\text {out }}$, we use these radial null geodesics to calculate the $\operatorname{Im} I$, as follows ${ }^{7}$

$$
\operatorname{Im} I=\operatorname{Im} \int_{r_{\text {in }}}^{r_{\text {out }}} p_{r} d r=\operatorname{Im} \int_{r_{\text {in }}}^{r_{\text {out }}} \int_{0}^{p_{r}} d p_{r}^{\prime} d r .
$$

Therefore, we incorporate back-reaction effects in a finite separation between initial and final radius as a result of self-gravitation effects of outgoing shells. On the other hand, according to energy conservation, the tunneling barrier is produced by a change in the

\footnotetext{
${ }^{7}$ Note that we require $r_{\text {in }}>r_{\text {out }}$ where $r_{\text {in }}=\frac{16 \pi G_{d} M}{(d-2) \Omega_{(d-2)}} \frac{1}{\Gamma\left(\frac{d-1}{2}\right)} \int_{0}^{\frac{r_{i n}^{2}}{4 \theta}} d t e^{-t} t^{\left(\frac{d-3}{2}\right)} \quad$ and $\quad r_{\text {out }}=$ $\frac{16 \pi G_{d}(M-E)}{(d-2) \Omega_{(d-2)}} \frac{1}{\Gamma\left(\frac{d-1}{2}\right)} \int_{0}^{\frac{r_{o u t}^{2}}{4 \theta}} d t e^{-t} t^{\left(\frac{d-3}{2}\right)}$.
} 
radius (reduction of black hole horizon) just by emitting particles. We now change the integration variable from momentum to energy by applying Hamilton's equation of motion, $\dot{r}=\left.\frac{d H}{d p_{r}}\right|_{r}$. So, we find

$$
\operatorname{Im} I=\operatorname{Im} \int_{M}^{M-E} \int_{r_{\text {in }}}^{r_{\text {out }}} \frac{d r}{\dot{r}} d H
$$

where the Hamiltonian is given by $H=M-E^{\prime}$. The $r$-integral can be solved firstly by deforming the contour so that

$$
\operatorname{Im} I=\operatorname{Im} \int_{0}^{E} \int_{r_{\text {in }}}^{r_{\text {out }}} \frac{d r}{1-\sqrt{\frac{2 \mathcal{M}_{\theta}\left(M-E^{\prime}\right)}{r^{d-3}}}}\left(-d E^{\prime}\right) .
$$

The $r$-integral has a pole at the horizon in which it lies on the line of integration and leads to $(-\pi i)$ times the residue

$$
\operatorname{Im} I=\operatorname{Im} \int_{0}^{E}(-\pi i)\left(\frac{2}{d-3}\right)\left(2 \mathcal{M}_{\theta}\left(M-E^{\prime}\right)\right)^{\frac{1}{d-3}}\left(-d E^{\prime}\right) .
$$

The above integration can be preformed without writing out the explicit form of radial null geodesics. In the vicinity of the horizon, $\dot{r}$ behaves as

$$
\dot{r} \simeq\left(r-r_{H}\right) \kappa(M)+O\left(\left(r-r_{H}\right)^{2}\right),
$$

where $\kappa(M)$ is the horizon surface gravity. The mentioned integration can be done simply by inserting the relation (3.10), in which self-gravitation is included, into the integral (3.7) and performing the $r$-integral as follows

$$
\operatorname{Im} I=-\operatorname{Im} \int_{0}^{E} \int_{r_{\text {in }}}^{r_{\text {out }}} \frac{d r d E^{\prime}}{\left(r-r_{H}\right) \kappa\left(M-E^{\prime}\right)}=\pi \int_{0}^{E} \frac{d E^{\prime}}{\kappa\left(M-E^{\prime}\right)} .
$$

Comparing two integrands in the relations (3.9) and (3.11) gives us

$$
\kappa(M-E)=\frac{d-3}{2\left(2 \mathcal{M}_{\theta}(M-E)\right)^{\frac{1}{d-3}}} .
$$

Now, Hawking temperature is given by

$$
T_{H}=\frac{\kappa(M)}{2 \pi} \approx \frac{d-3}{4 \pi\left(2 \mathcal{M}_{\theta}(M)\right)^{\frac{1}{d-3}}} .
$$

It is clear that, two Eqs. (2.16) and (3.13) for the NC black hole temperature coincide, but note that the approach to achieve (3.13) uses some approximations. This coincidence confirms that our calculations are approximately correct.

Here, we would like to obtain the radial geodesics of the massive particles which are different with massless case. Recently, some authors have extended Parikh-Wilczek work to the massive particles' tunneling [40, 41, 42]. In this regard, once again we follow the line element of the NC Painlevé-Schwarzschild black hole, that is

$$
d s^{2}=g_{00} d t^{2}+2 g_{01} d t d r+g_{11} d r^{2}+r^{2} d \Omega_{(d-2)}^{2} .
$$


This line-element can be obtained by definition of a noncommutative time coordinate transformation as

$$
d t=d t_{s}+d t_{s y n}
$$

where

$$
d t_{s y n}=\frac{\sqrt{2 \mathcal{M}_{\theta} r^{d-3}}}{r^{d-3}-2 \mathcal{M}_{\theta}} d r=-\frac{g_{01}}{g_{00}} d r
$$

This relation, which has been written based on the Landau's theory of the synchronization of clocks [43], allows us to synchronize clocks in any infinitesimal radial positions of particles $\left(d \Omega_{(d-2)}=0\right)$. Since the tunneling phenomena through the quantum horizon, the barrier, is an instantaneous procedure, it is important to apply Landau's theory of the coordinate clock synchronization to the tunneling process. The mechanism for tunneling through the quantum horizon is that particle anti-particle pair is created at the event horizon. So, we have two events that occur simultaneously; one event is anti-particle and tunnels into the barrier but the other particle tunnels out the barrier. In fact, the relation (3.16) mentions the difference of coordinate times for these two simultaneous events occurring at infinitesimally adjacent radial positions. According to the non-relativistic quantum theory, de Broglie's hypothesis and the WKB approximation, it can be easily shown that the treatment of the massive particle's tunneling as a massive shell is approximately derived by the phase velocity $v_{p}$ of the de Broglie s-wave. The relationship between phase velocity $v_{p}$ and group velocity $v_{g}$ is given by [40, 41, 42]

$$
v_{p}=\dot{r}=\frac{1}{2} v_{g}
$$

In the case of $d \Omega_{(d-2)}=0$, according to the relation $(3.16)$, the group velocity is

$$
v_{g}=-\frac{g_{00}}{g_{01}}
$$

Thus, the outgoing motion of the massive particles take the following form

$$
\dot{r}=-\frac{g_{00}}{2 g_{01}}=\frac{r^{d-3}-2 \mathcal{M}_{\theta}}{2 \sqrt{2 \mathcal{M}_{\theta} r^{d-3}}} .
$$

We should incorporate back-reaction effects in this relation and then the result should be inserted into relation (3.7) to obtain a relation corresponding to (3.9) but now for massive particles. Let us come back to the integral (3.9). This integral has not a closed analytical solution, so we are going to compute Taylor series expansion of integrand, with respect to the particle's energy $E$, about the zero point and just to first order. Then we can solve the integral to find

$$
\begin{aligned}
& \operatorname{Im} I=\pi\left[16 \pi G_{d} M \pi^{-\frac{d-1}{2}}\left(\frac{M^{2}}{\theta}\right)^{\frac{d-1}{2}} e^{-\frac{M^{2}}{\theta}}\left(4 M^{4} \mathcal{H}+2 d \theta M^{2}+6 \theta M^{2}+4 d \theta^{2}+3 \theta^{2}+d^{2} \theta^{2}\right)(d-2)^{-1}\right. \\
& \left.\theta^{-2}(d-1)^{-1}(1+d)^{-1}(d+3)^{-1}\right]^{\frac{1}{d-3}} E\left(-E d^{4} \theta^{3}-180 M^{3} \theta^{2}-90 M \theta^{3}-4 E M^{4} \mathcal{H} \theta d^{2}-16 E M^{6} \mathcal{H}^{\prime}+\right. \\
& 40 E M^{6} \mathcal{H}+2 M \theta^{3} d^{4}+4 M^{3} \theta^{2} d^{3}-120 M^{5} \theta \mathcal{H}+20 M^{3} \theta^{2} d^{2}-36 M^{3} \theta^{2} d+12 M \theta^{3} d^{3}-8 M \theta^{3} d^{2}+
\end{aligned}
$$




$$
\begin{aligned}
& 16 M^{5} \theta d \mathcal{H}+8 M^{5} \theta d^{2} \mathcal{H}+8 E M^{6} \mathcal{H} d-80 E M^{4} \mathcal{H} \theta-16 E d \theta^{2} M^{2}-2 E d^{2} \theta^{2} M^{2}+32 E \theta d M^{4}+ \\
& \left.4 E \theta d^{2} M^{4}-108 M \theta^{3} d+60 E \theta M^{4}-30 E \theta^{2} M^{2}-15 E d \theta^{3}-23 E d^{2} \theta^{3}-9 E d^{3} \theta^{3}-36 E M^{4} \mathcal{H} \theta d\right) \\
& (d-3)^{-2} \theta^{-1}(5+d)^{-1} M^{-1}\left(4 M^{4} \mathcal{H}+2 d \theta M^{2}+6 \theta M^{2}+4 d \theta^{2}+3 \theta^{2}+d^{2} \theta^{2}\right)^{-1}, \quad(3.20)
\end{aligned}
$$

where $\mathcal{H}=$ hypergeom $\left(1, \frac{5+d}{2}, \frac{M^{2}}{\theta}\right)$ and $\mathcal{H}^{\prime}=$ hypergeom $\left(2, \frac{7+d}{2}, \frac{M^{2}}{\theta}\right)$. Since, both particle and anti-particle (corresponding to a time reversed state that can be seen backward in time by replacing $\sqrt{\frac{2 \mathcal{M}_{\theta}}{r^{d-3}}}$ by $-\sqrt{\frac{2 \mathcal{M}_{\theta}}{r^{d-3}}}$ in the metric $(3.2)$ ) anticipate in the emission rate of the Hawking process via tunneling with the same amounts, we have to add their amplitudes firstly and then squaring it to obtain the emission probability (Eq. (3.5)).

On the other side, using the first law of black hole thermodynamics, $d M=\frac{\kappa}{2 \pi} d S$, and Eq. (3.11), one can find the imaginary part of the action as follows [2, 3, 4] (see also [44, 45, 46, 47])

$$
\operatorname{Im} I=-\frac{1}{2} \int_{S_{N C}(M)}^{S_{N C}(M-E)} d S=-\frac{1}{2} \Delta S_{N C},
$$

which shows that the tunneling approach concurs with the thermal Hawking spectrum just in the limit of low energy. Hawking radiation of black holes as quantum tunneling was also studied in the context of string theory [44], and it was demonstrated that the tunneling probability in the high energy limit is analogous to a difference between counting of states in the microcanonical and canonical ensembles. It can be easily seen that our computed tunneling probability to first order in $E$ generates the Boltzmann factor in the canonical ensemble $\Gamma \sim \exp (-\beta E)$, which is characterized by the inverse temperature as the coefficient $\beta$. So, the tunneling probabilities in the high energy limit are proportional to $\exp (\Delta S)$ (see also [48]),

$$
\Gamma \sim \exp (-2 \operatorname{Im} I) \sim \frac{e^{S_{\text {final }}}}{e^{S_{\text {initial }}}}=\exp (\Delta S)=\exp [S(M-E)-S(M)]
$$

where $\Delta S$ is the difference in black hole entropies before and after emission. This means that at higher energies the emission rate depends on the final and initial number of accessible microstates of the system. Therefore at higher energies the emission spectrum cannot be exactly thermal since the high energy modifications flow from the physics of energy conservation with noncommutativity corrections. In fact, to linear order in $E$, two expressions for $\Gamma$ in the microcanonical and canonical ensembles coincide. Thus, obviously the emission rate (3.22) deviates from the pure thermal emission but is corresponding to an underlying unitary quantum theory [3, 4].

At this stage, one can easily show that there are no correlations between emitted particles even with incorporation of the noncommutativity modifications in an extra dimensional setup. If there is any correlation between different emitted modes, at least part of the information coming out of the black hole to be retained in these correlations. As our analysis shows, at least up to second order in particle's energy, there will be no correlation between the different modes of radiation. This reflects the fact that information does not 
come out continuously during the evaporation process at least at late-times ${ }^{8}$. This indicates that the tunneling probability of two particles of energy $E_{1}$ and $E_{2}$ is comparable to the tunneling probability of one particle with composed energy, $E=E_{1}+E_{2}$, i.e.

$$
\Delta S_{E_{1}}+\Delta S_{E_{2}}=\Delta S_{\left(E_{1}+E_{2}\right)} \Rightarrow \chi\left(E_{1}+E_{2} ; E_{1}, E_{2}\right)=0
$$

It can be numerically confirmed that these probabilities of emission are actually uncorrelated. On the other hand, the statistical correlation function $\chi\left(E ; E_{1}, E_{2}\right)$, is zero which leads to the independence between different modes of radiation during the evaporation. Accordingly, space noncommutativity and higher dimensional considerations are not enough to provide the basis for existence of the correlations between different modes.

There are four main proposals about what happens to the information that falls into a black hole. Based on the first proposal, the black hole can evaporate completely as uncorrelated thermal radiation in each mode and all the information including the original quantum state that formed the black hole (excluding its mass, charge and angular momentum), would disappear from our universe. However, this proposal allows the pure states to evolve into the mixed states, which is incompatible with the basic principles of quantum mechanics. Second proposal is that the black hole can completely disappear, but the information appears in the final burst of radiation when the black hole shrinks to the Planck size. A third possibility is that the information comes out in non-thermal correlations within the Hawking radiation, the process being portrayed by a unitary $S$-matrix. In other words, there are non-thermal correlations between different modes of radiation throughout the evaporation process that information emerges ceaselessly encoded through them. And fourth idea is that the black hole never disappears completely, and the information is not lost, but would be stored in a Planckian-size stable remnant. In this section, we have studied the credibility of the third and fourth conjectures within a NC spacetime feature. We have shown that inclusion of noncommutativity and braneworld effects are not enough to create correlations between emitted modes of black hole evaporation. However, due to spacetime noncommutativity, the information might be preserved by a stable black hole remnant. In our opinion, this is one of the successful scenarios for information loss paradox currently [13]. In next section we introduce a procedure to create correlations between emitted modes.

\section{Quantum Gravity as a Source of Correlations}

All quantum gravity scenarios support the idea that there is a fundamental length scale that cannot be probed. For instance, in string theory there is a restraint in probing distances smaller than the string length. Hence the standard uncertainty principle is modified to include this restricted resolution of the spacetime points. The result of this modification is known as the Generalized Uncertainty Principle (GUP) which is actually a representation of the quantum nature of spacetime at Planck scale. In a model universe with extra

\footnotetext{
${ }^{8}$ Our calculations are performed based on Eq. (3.5) in stationary phase, hence the phrase late-time.
} 
dimensions, GUP can be generalized to the tensorial form as follows

$$
\Delta x_{i} \geq \frac{1}{\Delta p_{i}}+\alpha L_{P l}^{2} \Delta p_{i}
$$

where the fundamental Planck length is defined as $L_{P l}=G_{d}^{\frac{1}{d-2}}$ and $\alpha$ is a dimensionless constant of the order of unity that depends on the specific model. The above GUP can be acquired in the context of string theory [7], NC quantum theory [8], loop quantum gravity [9], or from black hole gedanken experiments [10]. In the standard limit, $\Delta x_{i} \gg L_{P l}$, it yields the ordinary uncertainty principle, $\Delta x_{i} \Delta p_{i} \geq 1$. The second term in r.h.s of Eq. (4.1) becomes considerable when the momentum and distance scales approach the Planck scale. For a spherical black hole the thermodynamic quantities can be obtained in a heuristic manner using the standard uncertainty principle [49. Application of the GUP to black hole thermodynamics in the same manner, modifies the results dramatically by incorporation of quantum gravity effects in the final stages of black hole evaporation with a rich phenomenology [16]. In this section we use GUP to find an alternative to retrieve the lost information in the black hole evaporation process. This alternative is related to the correlations between different modes of evaporation. Can GUP provide the required correlations? This is an important question which we answer in a tunneling framework. A first effort in this direction was given by Arzano et al [45], resulting to the quantum corrected entropy with an additional logarithmic correction term. However, such a formulation does not prepare a way to find a non-zero statistical correlation function. In this section we follow a different way to retrieve the information. In a recent paper [20], we investigated the modifications of the Hawking radiation by the tunneling process and the GUP. By using the GUP-corrected de Broglie wave length, the squeezing of the fundamental momentum-space cell, and consequently a GUP-corrected energy, we find the non-thermal effects which lead to a non-zero statistical correlation function between probabilities of tunneling of two particles with different energies. In this section, we extend our framework to model universe with extra dimensions.

Amelino-Camelia et al [50] (see also [51]) studied the black hole evaporation process after an analysis of the GUP-induced modification of the black body radiation spectrum. If GUP is fundamental concept of quantum gravity, it should appear in de Broglie relation as follows

or

$$
\lambda \simeq \frac{1}{p}\left(1+\alpha L_{P l}^{2} p^{2}\right)
$$

$$
\mathcal{E} \simeq E\left(1+\alpha L_{P l}^{2} E^{2}\right)
$$

There are other compelling reasons from NC geometry and loop quantum gravity that support relation (4.3) (see for instance [50, 51] and references therein). Now, we consider a massless particle i.e. a shell and take into consideration the response of the background geometry to a radiated quantum of energy $E$ with GUP correction i.e. $\mathcal{E}$. The particle moves on the geodesics of a spacetime with $M-\mathcal{E}$ substituted for $M$. With these preliminaries and also considering the deformed Hamilton's equation of motion as

$$
\left.\dot{r} \simeq\left(1+\alpha L_{P l}^{2} \mathcal{E}^{2}\right) \frac{d H}{d p_{r}}\right|_{r}
$$


eventually the imaginary part of the action takes the following form

$$
\operatorname{Im} I=\operatorname{Im} \int_{0}^{\mathcal{E}}(-\pi i)\left(\frac{2}{d-3}\right)\left(1+\alpha L_{P l}^{2} \mathcal{E}^{\prime 2}\right)\left(2\left(M-\mathcal{E}^{\prime}\right)\right)^{\frac{1}{d-3}}\left(-d \mathcal{E}^{\prime}\right) .
$$

The leading order correction is proportional to the square of $\left(\sqrt{\alpha} L_{p}\right)$. This integral, similar to the integral (3.9), has not a closed analytical solution, so we are going to compute Taylor series expansion of integrand (with respect to the particle's energy $E$ ) about the zero point and just to first order. Then we can solve the integral to find

$$
\begin{gathered}
\operatorname{Im} I=\frac{\pi}{(d-3)^{2}}\left[(d-3)\left(2^{d-2} M\right)^{\frac{1}{d-3}} E-\left(2 M^{4-d}\right)^{\frac{1}{d-3}} E^{2}+\right. \\
\left.+\alpha L_{P l}^{2} E^{3}\left((d-3)\left(2^{d-2} M\right)^{\frac{1}{d-3}}-2\left(2 M^{4-d}\right)^{\frac{1}{d-3}} E\right)+O\left(\alpha^{2} L_{P l}^{4}\right)\right] .
\end{gathered}
$$

The tunneling rate is therefore

$$
\begin{gathered}
\Gamma \sim \exp \left(-\frac{2 \pi}{(d-3)^{2}}\left[(d-3)\left(2^{d-2} M\right)^{\frac{1}{d-3}} E-\left(2 M^{4-d}\right)^{\frac{1}{d-3}} E^{2}+\right.\right. \\
\left.\left.+\alpha L_{P l}^{2} E^{3}\left((d-3)\left(2^{d-2} M\right)^{\frac{1}{d-3}}-2\left(2 M^{4-d}\right)^{\frac{1}{d-3}} E\right)+O\left(\alpha^{2} L_{P l}^{4}\right)\right]\right) .
\end{gathered}
$$

For $d=4$, the first and second expressions in the exponential exhibit non-thermal behavior that was discovered firstly in Ref. [2]. So, the emission spectrum cannot be strictly thermal. In our situation, there is an additional term depending on the GUP parameter in first order and cannot be neglected once the black hole mass becomes comparable to the Planck mass. We now illustrate whether or not, the emission rates for the different modes of radiation during the evaporation are mutually related from a statistical viewpoint. Using (4.7), the emission rate for a first quantum with energy $E_{1}$, gives

$$
\begin{gathered}
\ln \Gamma_{E_{1}}=-\frac{2 \pi}{(d-3)^{2}}\left[(d-3)\left(2^{d-2} M\right)^{\frac{1}{d-3}} E_{1}-\left(2 M^{4-d}\right)^{\frac{1}{d-3}} E_{1}^{2}+\right. \\
\left.+\alpha L_{P l}^{2} E_{1}^{3}\left((d-3)\left(2^{d-2} M\right)^{\frac{1}{d-3}}-2\left(2 M^{4-d}\right)^{\frac{1}{d-3}} E_{1}\right)\right] .
\end{gathered}
$$

Similarly, the emission rate for a second quantum $E_{2}$, takes the form

$$
\begin{gathered}
\ln \Gamma_{E_{2}}=-\frac{2 \pi}{(d-3)^{2}}\left[(d-3)\left(2^{d-2}\left(M-E_{1}\right)\right)^{\frac{1}{d-3}} E_{2}-\left(2\left(M-E_{1}\right)^{4-d}\right)^{\frac{1}{d-3}} E_{2}^{2}+\right. \\
\left.+\alpha L_{P l}^{2} E_{2}^{3}\left((d-3)\left(2^{d-2}\left(M-E_{1}\right)\right)^{\frac{1}{d-3}}-2\left(2\left(M-E_{1}\right)^{4-d}\right)^{\frac{1}{d-3}} E_{2}\right)\right] .
\end{gathered}
$$


Correspondingly, the emission rate for a single quantum with the same total energy, $E=$ $E_{1}+E_{2}$, yields

$$
\begin{gathered}
\ln \Gamma_{\left(E_{1}+E_{2}\right)}=-\frac{2 \pi}{(d-3)^{2}}\left[(d-3)\left(2^{d-2} M\right)^{\frac{1}{d-3}}\left(E_{1}+E_{2}\right)-\left(2 M^{4-d}\right)^{\frac{1}{d-3}}\left(E_{1}+E_{2}\right)^{2}+\right. \\
\left.+\alpha L_{P l}^{2}\left(E_{1}+E_{2}\right)^{3}\left((d-3)\left(2^{d-2} M\right)^{\frac{1}{d-3}}-2\left(2 M^{4-d}\right)^{\frac{1}{d-3}}\left(E_{1}+E_{2}\right)\right)\right] .
\end{gathered}
$$

It can be seen easily that these probabilities are correlated. On the other hand, the non-zero statistical correlation function is

$$
\begin{gathered}
\chi\left(E_{1}+E_{2} ; E_{1}, E_{2}\right)=\frac{2 \pi E_{2}}{(d-3)^{2}}\left[(d-3)\left[\left(2^{d-2}\left(M-E_{1}\right)\right)^{\frac{1}{d-3}}-\left(2^{d-2} M\right)^{\frac{1}{d-3}}\right]+\right. \\
+E_{2}\left[\left(2 M^{4-d}\right)^{\frac{1}{d-3}}-\left(2\left(M-E_{1}\right)^{4-d}\right)^{\frac{1}{d-3}}\right]+E_{1}\left(2^{d-2} M^{4-d}\right)^{\frac{1}{d-3}}+ \\
+\alpha L_{P l}^{2}\left(4 E_{1}\left(2 M^{4-d}\right)^{\frac{1}{d-3}}\left[2 E_{1}^{2}+2 E_{2}^{2}+3 E_{1} E_{2}\right]-(d-3)\left(2^{d-2} M\right)^{\frac{1}{d-3}}\left[3 E_{1}^{2}+E_{2}^{2}+3 E_{1} E_{2}\right]+\right. \\
\left.\left.+(d-3) E_{2}^{2}\left(2^{d-2}\left(M-E_{1}\right)\right)^{\frac{1}{d-3}}+E_{2}^{3}\left[\left(2^{d-2} M^{4-d}\right)^{\frac{1}{d-3}}-\left(2^{d-2}\left(M-E_{1}\right)^{4-d}\right)^{\frac{1}{d-3}}\right]\right)\right] .
\end{gathered}
$$

Note that three first terms in square bracket in r.h.s. can be neglected for any reasonable values of quantities. Appearance of these terms originates from approximations used in the calculation of integral in equation (4.5). Therefore we find

$$
\begin{gathered}
\chi\left(E_{1}+E_{2} ; E_{1}, E_{2}\right)=\frac{2 \pi \alpha L_{P l}^{2} E_{2}}{(d-3)^{2}}\left(4 E_{1}\left(2 M^{4-d}\right)^{\frac{1}{d-3}}\left[2 E_{1}^{2}+2 E_{2}^{2}+3 E_{1} E_{2}\right]-\right. \\
-(d-3)\left(2^{d-2} M\right)^{\frac{1}{d-3}}\left[3 E_{1}^{2}+E_{2}^{2}+3 E_{1} E_{2}\right]+(d-3) E_{2}^{2}\left(2^{d-2}\left(M-E_{1}\right)\right)^{\frac{1}{d-3}}+ \\
\left.+E_{2}^{3}\left[\left(2^{d-2} M^{4-d}\right)^{\frac{1}{d-3}}-\left(2^{d-2}\left(M-E_{1}\right)^{4-d}\right)^{\frac{1}{d-3}}\right]\right) .
\end{gathered}
$$

This means that not only the probability of tunneling of two particles of energy $E_{1}$ and $E_{2}$ is not the same as probability of tunneling of one particle with their compound energies, $E_{1}+E_{2}$, but also there are correlations between them. In fact, whenever one quantum of emission is radiated from the surface of the black hole horizon, the perturbations are created on the Planck scale that influence the second quantum of emission, and these perturbations cannot be neglected. Particularly, once the black hole mass becomes comparable with the Planck mass, one cannot neglect the effects of these perturbations. Therefore, as expected in Ref. [45], in this way the form of the amendments as back-reaction effects by incorporation of GUP effects, are sufficient by themselves to create correlation and therefore recovery of information. So, at least part of the information leaks out from the black hole as the non-thermal GUP correlations and this provides a basis to solve information loss problem. The question which arises here is time evolution of these correlations. This problem is currently under investigation. 


\section{Summary}

In this paper, generalization of the standard Hawking radiation via quantum tunneling through the smeared quantum horizon of the non-commutative Schwarzschild black hole has been studied. This study is based on the solution of the Eq. (3.5) in the context of coordinate coherent state noncommutativity and in a model universe with extra dimensions. The corrections of the emission rates for both massless and massive particles have been performed. We have shown that noncommutativity and extra-dimensional considerations are not enough to create correlations between emitted modes. That is, there are no correlations between the tunneling rates of the different modes in the black hole radiation spectrum at least at late-times and just to second order in E. However, spacetime noncommutativity is adequate by itself to preserve information due to the fact that in the NC framework, the black hole does not evaporate completely and this leads to the existence of a minimal non-zero mass (e.g., a Planck-sized remnant) in which black hole can reduce to it at final stage of evaporation. So, part of information might be preserved in this remnant. If one really believes the idea of stable black hole remnants due to the fact that there are some exact continuous global symmetries in the nature [52], then, based on our findings (Sections 2 and 3), we should accept this possibility that a part of information stays inside the black hole and can be retained by a stable Planck-sized remnant ${ }^{9}$. On the other hand, the generalized uncertainty principle of string theory or noncommutative quantum mechanics brings significant modifications to the Hawking radiation. As it is well-known, the GUP leads to a Planck-scale remnant at the endpoint of evaporation process. We have shown that the GUP as modification of the de Broglie relation in the quantum tunneling framework of the black hole evaporation, leads to correlation between emitted modes of evaporation. Hence in this setup, a part of the information leaks out of the black hole in the form of non-thermal GUP correlations, and the other part reside in the remnant. These features have the potential to answer some questions regarding the black hole information loss paradox and provide a more realistic background for treating the black hole evaporation process, especially in its final stages of evaporation. We stress that time-evolution of GUP-induced correlations is an important issue which needs further investigation and probably shed more light on information loss problem.

\section{Acknowledgments}

This work has been supported partially by Research Institute for Astronomy and Astrophysics of Maragha, Iran.

$*$

\section{A.}

The imaginary part of the action (Eq. (3.20)) with some arbitrary number of dimensions

\footnotetext{
${ }^{9}$ Of course, this assumption is acceptable if information is really conserved in our world.
} 
can be calculated as follows

$$
\begin{aligned}
& d=4 \longrightarrow \operatorname{Im} I=2 \sqrt{\pi} G_{4} e^{-\frac{M^{2}}{\theta}} E\left(-4 E \frac{M^{3}}{\theta^{\frac{3}{2}}}-4 \frac{M^{2}}{\sqrt{\theta}}+2 E \frac{M}{\sqrt{\theta}}+\sqrt{\pi} \mathcal{E}\left(\frac{M}{\sqrt{\theta}}\right) e^{\frac{M^{2}}{\theta}}[2 M-E]\right) \\
& d=5 \longrightarrow \operatorname{Im} I=\left[\frac{\pi}{6} G_{5} M e^{-\frac{M^{2}}{\theta}}\left(-\frac{M^{2}}{\theta}-1+e^{\frac{M^{2}}{\theta}}\right)\right]^{\frac{1}{2}}\left(M^{3} \theta e^{-\frac{M^{2}}{\theta}}+M \theta^{2} e^{-\frac{M^{2}}{\theta}}-M \theta^{2}\right)^{-1} \\
& E e^{-\frac{M^{2}}{\theta}}\left(2 E M^{4}+4 M^{3} \theta-E \theta M^{2}+4 M \theta^{2}-4 M \theta e^{\frac{M^{2}}{\theta}}-E \theta^{2}+E \theta^{2} e^{\frac{M^{2}}{\theta}}\right) \\
& d=6 \longrightarrow \operatorname{Im} I=\frac{\sqrt{\pi}}{18} 2^{\frac{2}{3}}\left(G_{6} M e^{-\frac{M^{2}}{\theta}}\left(-4 \frac{M^{3}}{\theta^{\frac{3}{2}}}-6 \frac{M}{\sqrt{\theta}}+3 \sqrt{\pi} \mathcal{E}\left(\frac{M}{\sqrt{\theta}}\right) e^{\frac{M^{2}}{\theta}}\right)\right)^{\frac{1}{3}} \\
& E\left(-8 E \frac{M^{5}}{\sqrt{\theta}}-24 M^{4} \sqrt{\theta}+4 E M^{3} \sqrt{\theta}-36 M^{2} \theta^{\frac{3}{2}}+6 E M \theta^{\frac{3}{2}}+\sqrt{\pi} \theta^{2} \mathcal{E}\left(\frac{M}{\sqrt{\theta}}\right) e^{\frac{M^{2}}{\theta}}[18 M-3 E]\right) \\
& \left(-4 M^{4} \sqrt{\theta}-6 M^{2} \theta^{\frac{3}{2}}+3 M \sqrt{\pi} \theta^{2} \mathcal{E}\left(\frac{M}{\sqrt{\theta}}\right) e^{\frac{M^{2}}{\theta}}\right)^{-1}
\end{aligned}
$$

and so on.

\section{References}

[1] S. W. Hawking, Particle creation by black holes, Commun. Math. Phys. 43 (1975) 199.

[2] M. K. Parikh and F. Wilczek, Hawking radiation as tunneling, Phys. Rev. Lett. 85 (2000) 5042 arXiv:hep-th/9907001.

[3] M. K. Parikh, A Secret Tunnel Through The Horizon, Int. J. Mod. Phys. D 13 (2004) 2351 arXiv:hep-th/0405160].

[4] M. K. Parikh, Energy Conservation and Hawking Radiation, hep-th/0402166.

[5] P. Nicolini, A. Smailagic and E. Spallucci, Noncommutative geometry inspired Schwarzschild black hole, Phys. Lett. B 632 (2006) 547 arXiv:gr-qc/0510112.

[6] P. Nicolini, Noncommutative Black Holes, The Final Appeal To Quantum Gravity: A Review, arXiv:0807.1939.

[7] D. Amati, M. Ciafaloni and G. Veneziano, Can spacetime be probed below the string size?, Phys. Lett. B 216 (1989) 41; K. Konishi, G. Paffuti and P. Provero, Minimum physical length and the generalized uncertainty principle in string theory, Phys. Lett. B 234 (1990) 276 .

[8] M. Maggiore, Quantum Groups, Gravity, and the Generalized Uncertainty Principle, Phys. Rev. D 49 (1994) 5182 arXiv:hep-th/9305163]; and A Generalized Uncertainty Principle in Quantum Gravity, Phys. Lett. B 304 (1993) 65 arXiv:hep-th/9301067; and The algebraic structure of the generalized uncertainty principle, Phys. Lett. B 319 (1993) 83 arXiv:hep-th/9309034.

[9] L. J. Garay, Quantum Gravity and Minimum Length, Int. J. Mod. Phys. A 10 (1995) 145 arXiv:gr-qc/9403008. 
[10] F. Scardigli, Generalized Uncertainty Principle in Quantum Gravity from Micro-Black Hole Gedanken Experiment, Phys. Lett. B 452 (1999) 39 arXiv:hep-th/9904025.

[11] A. Kempf, G. Mangano and R. B. Mann, Hilbert space representation of the minimal length uncertainty relation, Phys. Rev. D 52 (1995) 1108 arXiv:hep-th/9412167; H. Hinrichsen and A. Kempf, Maximal Localisation in the Presence of Minimal Uncertainties in Positions and Momenta, J. Math. Phys. 37 (1996) 2121 arXiv: hep-th/9510144; K. Nozari, Some Aspects of Planck Scale Quantum Optics, Phys. Lett. B 629 (2005) 41 arXiv:hep-th/0508078; K. Nozari and T. Azizi, Some Aspects of Minimal Length Quantum Mechanics, Gen. Rel. Grav. 38 (2006) 735 arXiv:quant-ph/0507018; K. Nozari and S. H. Mehdipour, Implications of Minimal Length Scale on the Statistical Mechanics of Ideal Gas, Chaos, Solitons and Fractals 32 (2007) 1637 arXiv:hep-th/0601096; S.

Hossenfelder, Interpretation of Quantum Field Theories with a Minimal Length Scale, Phys. Rev. D 73 (2006) 105013 arXiv:hep-th/0603032]; and Self-consistency in Theories with a Minimal Length, Class. and Quant. Grav. 23 (2006) 1815 arXiv:hep-th/0510245; S.

Hossenfelder et al, Signature in the Planck Regime, Phys. Lett. B 575 (2003) 85 arXiv:hep-th/0305262.

[12] A. Smailagic and E. Spallucci, Feynman path integral on the non-commutative plane, J. Phys. A 36 (2003) L467 arXiv:hep-th/0307217; and UV divergence-free QFT on noncommutative plane, J. Phys. A 36 (2003) L517 arXiv:hep-th/0308193; and Lorentz invariance, unitarity and UV-finiteness of QFT on noncommutative spacetime, J. Phys. A 37 (2004) 7169 arXiv:hep-th/0406174]; E. Spallucci, A. Smailagic and P. Nicolini, Trace Anomaly in Quantum Spacetime Manifold, Phys. Rev. D 73 (2006) 084004 arXiv:hep-th/0604094.

[13] K. Nozari and S. H. Mehdipour, Hawking Radiation as Quantum Tunneling from a Noncommutative Schwarzschild Black Hole, Class. and Quant. Grav. 25 (2008) 175015 arXiv:0801.4074.

[14] J. Preskill, Do Black Holes Destroy Information?, hep-th/9209058; D. N. Page, Information in black hole radiation, Phys. Rev. Lett. 71 (1993) 3743 arXiv:hep-th/9306083; C. R. Stephens, G. 't Hooft and B. F. Whiting, Black hole evaporation without information loss, Class. and Quant. Grav. 11 (1994) 621 arXiv:gr-qc/9310006; A. Strominger, Les Houches Lectures on Black Holes, hep-th/9501071; T. Banks, Lectures on black holes and information loss, Nucl. Phys. 41 (Proc. Suppl.) (1995) 21 arXiv:hep-th/9412131; J. G. Russo, hep-th/0501132 and references therein.

[15] There is a large literature on this subject; see for example, C. Castro, String Theory, Scale Relativity and the Generalized Uncertainty Principle, Found. Phys. Lett. 10 (1997) 273, arXiv:hep-th/9512044; S. Capozziello, G. Lambiase and G. Scarpe, Generalized Uncertainty Principle from Quantum Geometry, Int. J. Theor. Phys. 39 (2000) 15 arXiv:gr-qc/9910017; S. K. Rama, Some Consequences of the Generalised Uncertainty Principle: Statistical Mechanical, Cosmological, and Varying Speed of Light, Phys. Lett. B 519 (2001) 103 arXiv:hep-th/0107255; A. Camacho, Generalized Uncertainty Principle and deformed dispersion relation induced by nonconformal metric fluctuations, Gen. Rel. Grav. 34 (2002) 1839 arXiv:gr-qc/0206006; F. Nasseri, Corrections to the Fine Structure Constant in D-dimensional Space from the Generalized Uncertainty Principle, Phys. Lett. $\mathbf{B}$ 618 (2005) 229 arXiv: astro-ph/0208222; M. Cavaglià and S. Das, How classical are TeV-scale black holes?, Class. and Quant. Grav. 21 (2004) 4511 [arXiv:hep-th/0404050; M. R. Setare, Corrections to the Cardy-Verlinde formula from the generalized uncertainty 
principle, Phys. Rev. D 70 (2004) 087501 arXiv:hep-th/0410044; B. Bolen and M. Cavaglià, (Anti-)de Sitter Black Hole Thermodynamics and the Generalized Uncertainty Principle, Gen. Rel. Grav. 37 (2005) 1255 arXiv:gr-qc/0411086; K. Nozari and S. H. Mehdipour, Wave packets propagation in quantum gravity, Gen. Rel. Grav. 37 (2005) 1995 arXiv:quant-ph/0507019; K. Nozari and S. H. Mehdipour, Black hole Remnants in Extra Dimensions and Dark Matter, Int. J. Mod. Phys. A 21 (2006) 4979

arXiv:gr-qc/0511110]; B. Vakili, Dilaton Cosmology, Noncommutativity and Generalized Uncertainty Principle, Phys. Rev. D 77 (2008) 044023 arXiv:0801.2438]; K. Nozari and S. H. Mehdipour, Gravitational Uncertainty and Black Hole Remnants, Mod. Phys. Lett. A 20 (2005) 2937 arXiv:0809.3144.

[16] R. J. Adler, P. Chen, D. I. Santiago, The Generalized Uncertainty Principle and Black Hole Remnants, Gen. Rel. Grav. 33 (2001) 2101 arXiv:gr-qc/0106080; A. J. M. Medved and E. C. Vagenas, When conceptual worlds collide: The GUP and the BH entropy, Phys. Rev. D 70 (2004) 124021 arXiv: hep-th/0411022.

[17] R. Gambini and J. Pullin, Nonstandard optics from quantum spacetime, Phys. Rev. D 59 (1999) 124021 arXiv:gr-qc/9809038.

[18] J. Alfaro, H. A. Morales-Tecotl and L. F. Urrutia, Quantum gravity corrections to neutrino propagation, Phys. Rev. Lett. 84 (2000) 2318 arXiv:gr-qc/9909079.

[19] G. Amelino-Camelia, M. Arzano and A. Procaccini, Severe constraints on Loop-Quantum-Gravity energy-momentum dispersion relation from black-hole area-entropy law, Phys. Rev. D 70 (2004) 107501 arXiv:gr-qc/0405084; and A glimpse at the flat-spacetime limit of quantum gravity using the Bekenstein argument in reverse, Int. J. Mod. Phys. D 13 (2004) 2337 arXiv:hep-th/0506182.

[20] K. Nozari and S. H. Mehdipour, Quantum gravity and recovery of information in black hole evaporation, Europhys. Lett. 84 (2008) 20008 [arXiv:0804.4221.

[21] A. Connes, Non-commutative Geometry, (Academic Press, New York, 1994); N. Seiberg and E. Witten, String theory and noncommutative geometry, J. High Energy Phys. 9909 (1999) 032 arXiv:hep-th/9908142; M. R. Douglas and N. A. Nekrasov, Noncommutative field theory, Rev. Mod. Phys. 73 (2001) 977 arXiv:hep-th/0106048]; A. Micu and M. M. Sheikh-Jabbari, Noncommutative $\Phi^{4}$ Theory at Two Loops, J. High Energy Phys. 0101 (2001) 025 arXiv:hep-th/0008057; M. Chaichian, P. Presnajder, M. M. Sheikh-Jabbari and A. Tureanu, Noncommutative Standard Model: Model Building, Eur. Phys. J. C 29 (2003) 413 arXiv:hep-th/0107055.

[22] I. Hinchliffe, N. Kersting and Y. L. Ma, Review of the Phenomenology of Noncommutative Geometry, Int. J. Mod. Phys. A 19 (2004) 179 arXiv:hep-ph/0205040 and references therein.

[23] J. L. Hewett, F. J. Petriello and T. G. Rizzo, Noncommutativity and unitarity violation in gauge boson scattering, Phys. Rev. D 66 (2002) 036001 arXiv:hep-ph/0112003; J. M. Conroy, H. J. Kwee and V. Nazaryan, Phenomenology of Lorentz-conserving noncommutative QED, Phys. Rev. D 68 (2003) 054004 arXiv:hep-ph/0305225; T. Ohl and J. Reuter, Testing the noncommutative standard model at a future photon collider, Phys. Rev. D 70 (2004) 076007 arXiv:hep-ph/0406098; B. Melic, K. Passek-Kumericki, J. Trampetic, P. Schupp and M. Wohlgenannt, The standard model on non-commutative space-time: strong interactions included, Eur. Phys. J. C 42 (2005) 499 arXiv:hep-ph/0503064; P. Schupp, J. Trampetic, J. Wess and G. Raffelt, The 
photon-neutrino interaction induced by non-commutativity and astrophysical bounds, Eur. Phys. J. C 36 (2004) 405 arXiv: hep-ph/0212292.

[24] I. Antoniadis, A possible new dimension at a few TeV Phys. Lett. B 246 (1990) 377; J. D. Lykken, Weak scale superstrings, Phys. Rev. D 54 (1996) R3693 arXiv:hep-th/9603133; E. Witten, Strong Coupling Expansion Of Calabi-Yau Compactification, Nucl. Phys. B 471 (1996) 135 arXiv:hep-th/9602070.

[25] N. Arkani-Hamed, S. Dimopoulos and G. Dvali, The hierarchy problem and new dimensions at a millimeter, Phys. Lett. B 429 (1998) 263 arXiv:hep-ph/9803315; I. Antoniadis, N. Arkani-Hamed, S. Dimopoulos and G. Dvali, New Dimensions at a Millimeter to a Fermi and Superstrings at a TeV, Phys. Lett. B 436 (1998) 257 arXiv:hep-ph/9804398; L. Randall and R. Sundrum, A Large Mass Hierarchy from a Small Extra Dimension, Phys. Rev. Lett. 83 (1999) 3370 arXiv:hep-ph/9905221 and An Alternative to Compactification , Phys. Rev. Lett. 83 (1999) 4690 [arXiv: hep-th/9906064.

[26] J. E. Moyal, Quantum mechanics as a statistical theory, Proc. Cambridge Phil. Soc. 45 (1949) 99.

[27] X. Calmet and A. Kobakhidze, Noncommutative general relativity, Phys. Rev. D 72 (2005) 045010 arXiv:hep-th/0506157; and Second order noncommutative corrections to gravity, Phys. Rev. D 74 (2006) 047702 [arXiv:hep-th/0605275]; A. H. Chamseddine, Deforming Einstein's gravity, Phys. Lett. B 504 (2001) 33 [arXiv:hep-th/0009153; P. Aschieri et al, A gravity theory on noncommutative spaces, Class. and Quant. Grav. 22 (2005) 3511 arXiv:hep-th/0504183.

[28] A. Gruppuso, Newton's law in an effective non-commutative space time, J. Phys. A 38 (2005) 2039 arXiv:hep-th/0502144.

[29] T. G. Rizzo, Noncommutative inspired black holes in extra dimensions, J. High Energy Phys. 0609 (2006) 021 arXiv:hep-ph/0606051.

[30] S. B. Giddings and S. Thomas, High energy colliders as black hole factories: The end of short distance physics, Phys. Rev. D 65 (2002) 056010 arXiv:hep-ph/0106219.

[31] K. Nozari and S. H. Mehdipour, Chaos, Solitons and Fractals (In Press), hep-th/0610076.

[32] S. Ansoldi, P. Nicolini, A. Smailagic and E. Spallucci, Noncommutative geometry inspired charged black holes, Phys. Lett. B 645 (2007) 261 arXiv:gr-qc/0612035.

[33] E. Spallucci, A. Smailagic and P. Nicolini, Pair creation by higher dimensional, regular, charged, micro black holes, arXiv:0801.3519.

[34] P. C. Argyres, S. Dimopoulos, and J. March-Russell, Black holes and sub-millimeter dimensions, Phys. Lett. B 441 (1998) 96 arXiv:hep-th/9808138; R. Emparan, G. T. Horowitz and R. C. Myers, Black Holes Radiate Mainly on the Brane, Phys. Rev. Lett. 85 (2000) 499 arXiv: hep-th/0003118; V. Cardoso, E. Berti and M. Cavaglià, LETTER TO THE EDITOR: What we (don't) know about black-hole formation in high-energy collisions, Class. and Quant. Grav. 22 (2005) L61 arXiv:hep-ph/0505125.

[35] K. Nozari and B. Fazlpour, Thermodynamics of Noncommutative Schwarzschild Black Hole, Mod. Phys. Lett. A 22 (2007) 2917 arXiv:hep-th/0605109.

[36] M. Chaichian, M. M. Sheikh-Jabbari and A. Tureanu, Hydrogen Atom Spectrum and the Lamb Shift in Noncommutative QED, Phys. Rev. Lett. 86 (2001) 2716 arXiv:hep-th/0010175. 
[37] P. Painlevé, La mécanique classique et la théorie de la relativité, Compt. Rend. Acad. Sci. (Paris) 173 (1921) 677.

[38] P. Kraus and F. Wilczek, Self-Interaction Correction to Black Hole Radiance, Nucl. Phys. B 433 (1995) 403 arXiv:gr-qc/9408003; and Some Applications of a Simple Stationary Line Element for the Schwarzschild Geometry, Mod. Phys. Lett. A 40 (1994) 3713 arXiv: gr-qc/9406042.

[39] S. Shankaranarayanan, T. Padmanabhan and K. Srinivasan, Hawking radiation in different coordinate settings: complex paths approach, Class. and Quant. Grav. 19 (2002) 2671 arXiv:gr-qc/0010042; and Method of complex paths and general covariance of Hawking radiation, Mod. Phys. Lett. A 16 (2001) 571 arXiv:gr-qc/0007022.

[40] J. Zhang and Z. Zhao, Massive particles' black hole tunneling and de Sitter tunneling, Nucl. Phys. B 725 (2005) 173 .

[41] J. Zhang and Z. Zhao, Hawking radiation of charged particles via tunneling from the Reissner-Nordstrøm black hole, J. High Energy Phys. 0510 (2005) 055.

[42] Q. Q. Jiang, S. Q. Wu and X. Cai, Hawking radiation as tunneling from the Kerr and Kerr-Newman black holes, Phys. Rev. D 73 (2006) 064003 arXiv:hep-th/0512351.

[43] L. D. Landau and E. M. Lifshitz, The classical theory of field, Pergamon Press, London, 1975.

[44] E. Keski-Vakkuri and P. Kraus, Microcanonical D-branes and Back Reaction, Nucl. Phys. B 491 (1997) 249 arXiv:hep-th/9610045.

[45] M. Arzano, A. Medved and E. Vagenas, Hawking Radiation as Tunneling through the Quantum Horizon, J. High Energy Phys. 0509 (2005) 037 arXiv: hep-th/0505266; A. J.

M. Medved and E. Vagenas, On Hawking Radiation as Tunneling with Logarithmic Corrections, Mod. Phys. Lett. A 20 (2005) 1723 arXiv:gr-qc/0505015; and On Hawking Radiation as Tunneling with Back-Reaction, Mod. Phys. Lett. A 20 (2005) 2449 arXiv:gr-qc/0504113.

[46] R. Banerjee, B. R. Majhi and S. Samanta, Noncommutative Black Hole Thermodynamics, Phys. Rev. D 77 (2008) 124035 arXiv:0801.3583; R. Banerjee, B. R. Majhi and S. K. Modak, Area Law in Noncommutative Schwarzschild Black Hole, arXiv:0802.2176.

[47] R. Banerjee and B. R. Majhi, Quantum Tunneling and Back Reaction, Phys. Lett. B 662 (2008) 62 arXiv:0801.0200; and Quantum Tunneling Beyond Semiclassical Approximation, J. High Energy Phys. 06 (2008) 095 arXiv:0805.2220; and Quantum Tunneling and Trace Anomaly, arXiv:0808.3688.

[48] S. Massar and R. Parentani, How the Change in Horizon Area Drives Black Hole Evaporation, Nucl. Phys. B 575 (2000) 333 arXiv:gr-qc/9903027.

[49] H. Ohanian and R. Ruffini, Gravitation and Spacetime, 2nd ed., p. 481 (W. W. Norton, 1994).

[50] G. Amelino-Camelia, M. Arzano, Y. Ling and G. Mandanici, Black-hole thermodynamics with modified dispersion relations and generalized uncertainty principles, Class. and Quant. Grav. 23 (2006) 2585 arXiv:gr-qc/0506110.

[51] K. Nozari and A. S. Sefidgar, Comparison of Approaches to Quantum Correction of Black Hole Thermodynamics, Phys. Lett. B 635 (2006) 156 arXiv:gr-qc/0601116. 
[52] J. D. Bekenstein, Nonexistence of baryon number for static black holes, Phys. Rev. D 5 (1972) 1239 